\title{
Interaction of miconazole oral gel with warfarin and cyclosporine in a patient with nephrotic syndrome
}

\author{
Reiko Tajima-Okubo $\cdot$ Shuichi Tsuruoka $\cdot$ \\ Noriaki Moriyama $\cdot$ Tae Kaneda $\cdot$ Kunihiro Yamagata
}

Received: 18 January 2012/ Accepted: 2 April 2012/Published online: 28 April 2012

(C) Japanese Society of Nephrology 2012

\begin{abstract}
It is well known that miconazole inhibits cytochrome P450 (CYP). However, this drug in oral gel form is believed not to absorb into systemic circulation, and therefore not to inhibit CYP. We experienced a case of increased oral bioavailability of warfarin and cyclosporine with this gel for treatment of oral candidiasis in a patient with nephrotic syndrome. Her prothrombin time-international normalized ratio (PT-INR) increased from 2 to 7.25 , and the cyclosporine concentration at $2 \mathrm{~h}$ after final dosing (C2) increased from 633.8 to $1396.5 \mathrm{ng} / \mathrm{ml} 6$ days after the coadministration. These problems were resolved by termination of the gel and switching to amphotericin B gargle. We further detected a significant amount of miconazole in her plasma, directly showing for the first time in a patient with this interaction that oral miconazole gel was actually absorbed into systemic circulation. Because warfarin and cyclosporine are often used simultaneously by nephrologists, caution should be applied with combined use of these drugs and oral miconazole gel to avoid adverse reaction.
\end{abstract}

R. Tajima-Okubo · S. Tsuruoka $(\bowtie) \cdot$ N. Moriyama .

K. Yamagata

Department of Nephrology, Faculty of Medicine,

University of Tsukuba,

1-1-1 Tennodai, Tsukuba, Ibaraki 305-8575, Japan

e-mail: tsuru@md.tsukuba.ac.jp

T. Kaneda

Department of Pharmacy, Tsukuba University Hospital,

Tsukuba, Japan
Keywords Antifungal drug - Cytochrome P450 . Pharmacokinetic drug interaction · Warfarin

\section{Background}

Warfarin and cyclosporine are often used for treatment of nephrotic syndrome as anticoagulant and immunosuppressant, respectively. It is well known that these drugs are metabolized by cytochromes P450(CYP)2C9 and CYP3A4, respectively [1]. Therefore, coadministration of drugs that alter CYP2C9 and CYP3A4 activity affects the efficacy and toxicity of warfarin and cyclosporine, respectively. Antifungal imidazole derivatives including miconazole are used systematically and topically for treatment of candidiasis. Their systemic administration inhibits CYP activity by inhibiting heme-binding site of the protein [2]. Thus, systemic miconazole administration increases bioavailability of CYP substrates, some of which can be life threatening. Several forms of topical agents, including oral gel, are clinically available for miconazole. These are believed to act locally and not be absorbed into systemic circulation, which is beneficial to avoid adverse reactions. Several reports state that oral miconazole gel increased the bioavailability of substrates of CYPs including warfarin [3, 4], which suggests systemic absorption of the gel. However, no direct evidence of absorption during the interaction was reported [4]. We experienced a patient with nephrotic syndrome whose prothrombin time-international normalized ratio (PT-INR) and cyclosporine exposure rapidly increased following coadministration of oral miconazole gel for oral candidiasis. We detected a significant amount of miconazole in her plasma, directly showing for the first time in a patient with this interaction that oral miconazole gel was actually 
absorbed into systemic circulation and caused increased systemic exposure of both warfarin and cyclosporine.

\section{Case report}

A 71-year-old woman was admitted to our hospital because of massive proteinuria (10 g/day) and systemic edema. She was clinically diagnosed with minimal change disease and entered complete remission with oral prednisolone $(40 \mathrm{mg} /$ day) and cyclosporine (150 mg/day). During the course, she was also treated with warfarin (2-2.5 mg/day) to avoid hypercoagulability, as well as furosemide (60 mg/day), spironolactone (25 mg/day), lansoprazole (15 mg/day), and sulfamethoxazole/trimethoprim (400 mg, twice weekly). Her renal function was moderately disturbed (serum creatinine concentration $1.5 \mathrm{mg} / \mathrm{dl}$ ). Her PT-INR was well controlled between 1.0 and 2.0 by $2-2.5 \mathrm{mg} / \mathrm{day}$ warfarin. However, her PT-INR rapidly rose to 7.25 on 1 November 2010 (Fig. 1). She had suffered from oral candidiasis and was prescribed $400 \mathrm{mg} /$ day miconazole oral gel (Florid oral gel ${ }^{\circledR}$; Mochida Pharmaceuticals, Japan) four times daily from 7 days before the episode. Vitamin $\mathrm{K}$ was administered and warfarin was stopped while miconazole was continued. Because INR decreased to 1.46, $2 \mathrm{mg} /$ day warfarin was restarted the next day. However, INR increased again to 6.276 days after the restart. Because we suspected an interaction between them, both drugs were stopped and amphotericin B gargle was started. One week later, warfarin was reintroduced and INR was well controlled at around 2.0 at the previous dose.

We routinely monitored her whole-blood cyclosporine concentration. Just before miconazole gel initiation on October 25, trough concentration (C0) was $132.9 \mathrm{ng} / \mathrm{ml}$, and $633.8 \mathrm{ng} / \mathrm{ml} 2 \mathrm{~h}$ after administration (C2). When we found the first INR increase on November 1, it had increased to $142.8 \mathrm{ng} / \mathrm{ml}$ at $\mathrm{C} 0$ and $1396.5 \mathrm{ng} / \mathrm{ml}$ at $\mathrm{C} 2$. Therefore, we reduced the dose to $125 \mathrm{mg} / \mathrm{day}$, and its blood concentration decreased to the previous level. After switching from miconazole gel to amphotericin B gargle, cyclosporine A ( $\mathrm{CsA}$ ) concentration was decreased, so we escalated to $150 \mathrm{mg}$ /day to obtain a similar trough level about 3 weeks later. We further measured plasma miconazole concentration by high-performance liquid chromatography (HPLC) [5]. The detection limit of the assay was $0.04 \mu \mathrm{g} / \mathrm{ml}$. We found that the concentrations before and $2 \mathrm{~h}$ after final dosing on November 1 were 0.13 and $0.35 \mu \mathrm{g} / \mathrm{ml}$, respectively. Thus, a significant amount of miconazole was detected in her plasma, which showed for the first time in a patient with this interaction that oral miconazole gel was actually absorbed into systemic circulation and caused increased systemic exposure of both warfarin and cyclosporine.

\section{Discussion}

We experienced a case of increased oral bioavailability of two drugs (i.e., warfarin and cyclosporine) with miconazole oral gel in a patient with nephrotic syndrome. There are several case reports of interaction of miconazole oral gel and warfarin in various diseases [3, 4]; however, our case is the first to show increased bioavailability of two drugs simultaneously. Furthermore, although these reports suggested absorption of miconazole into the body, direct evidence of absorption by measurement of the drug concentration in patient plasma was not reported. We detected a significant amount of the drug in her plasma and
Fig. 1 Clinical course of the patient. Ordinate represents dose of warfarin (mg/day) and value of PT-INR. $M C Z$ miconazole oral gel, $A M P H-B$ amphotericin B gargle

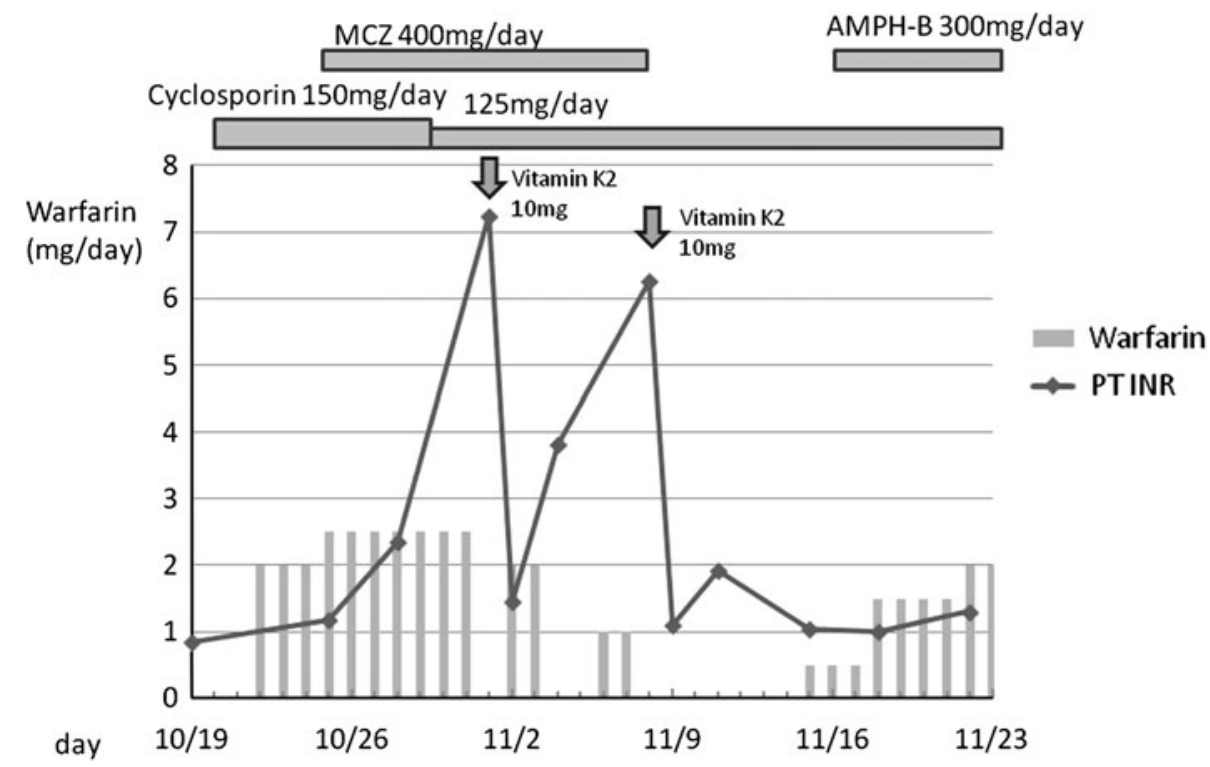


proved the hypothesis for the first time, which we believe to be the important point of this case.

According to drug interaction study with healthy volunteers taking miconazole tablets and warfarin, the trough concentration of plasma miconazole during pharmacokinetic interaction was 3-26 ng/ml [6], which was lower than in our patient. Therefore, the plasma concentration observed in our patient seems sufficient to cause the interaction. The increase of INR occurred 1-2 weeks after initiation of treatment with miconazole in previous report [6], which is compatible with the present case. Pharmacokinetic study with healthy volunteers also showed that the maximum plasma concentration of miconazole following repeated dosing of $125 \mathrm{mg}$ oral gel formula was $0.4-1.0 \mu \mathrm{g} / \mathrm{ml}$ [7], which is comparable to our patient's result. We also found reduced bioavailability of CsA after termination of miconazole oral gel. Therefore, we think the interaction between CsA and warfarin is minor, at least clinically.

The mechanisms concerning the high drug absorption in our patient are unclear. One possibility is absorption from intestine after swallowing. Oral miconazole gel dissolves in saliva [7]. Therefore, miconazole in swallowed oral gel and saliva might be absorbed from intestine. Another possibility is absorption from oral mucosa. Because this drug was used to treat oral candidiasis, the oral cavity infection may have altered absorption via mucosal barrier. The patient's moderate renal dysfunction may have reduced elimination of metabolites into urine. Further pharmacokinetic study with patients with oral cavity infection is needed. Because warfarin and cyclosporine are often used simultaneously by nephrologists, caution should be applied with combined use of these drugs and oral miconazole gel to avoid adverse reaction.

Conflict of interest All authors report no conflicts of interest.

\section{References}

1. Quinn D, Day R. Clinically important drug interactions. In: Speight T, Holford N, editors. Avery's drug treatment. Auckland: Adis International; 1997. p. 302-37.

2. Edwards I. Pharmacological basis of adverse drug reactions. In: Speight T, Holford N, editors. Avery's drug treatment. Auckland: Adis International; 1997. p. 261-99.

3. Colquhoun M, Daly M, Stewart P, Beeley L. Interaction between warfarin and miconazole oral gel. Lancet. 1987;i:695-6.

4. Pemberton MN, Oliver R, Theaker E. Miconazole oral gel and drug interactions. Br Dent J. 2004;196:529-31.

5. Kobylińska M, Kobylińska K, Sobik B. High-performance liquid chromatographic analysis for the determination of miconazole in human plasma using solid-phase extraction. J Chromatogr B Biomed Appl. 1996;685:191-5.

6. O'Reilly R, Goulart D, Kunze K, Neal J, Gibaldi M, Eddy A, et al. Mechanisms of the stereoselective interaction between miconazole and racemic warfarin in human subjects. Clin Pharmacol Ther. 1992;51:656-67.

7. Cardot J, Chaumont C, Dubray C, Costantini D, Aiache J. Comparison of the pharmacokinetics of miconazole after administration via a bioadhesive slow release tablet and an oral gel to healthy male and female subjects. $\mathrm{Br} \mathrm{J}$ Clin Pharmacol. 2004;58:345-51. 